\title{
Pump Power Reduction by Photodarkening in Yb-doped Fibres
}

\author{
S. $\mathrm{Yoo}^{1}, \boldsymbol{N} . L i^{2}, X . \mathrm{Yu}^{2}, \mathrm{~J} . \mathrm{K} . \mathrm{Sahu} \mathbf{u}^{3}$ \\ 1. School of Electrical and Electronic Engineering, Nanyang Technological University, 50 Nanyang Avenue, Singapore 639798, Singapore \\ 2. Singapore Institute of Manufacturing Technology, 71 Nanyang Drive, Singapore 638075, Singapore \\ 3. Optoelectronic Research Centre, University of Southampton, Southampton SO17 1BJ, U.K.
}

Photodarkening can be created under pumping at the $\mathrm{Yb}$ absorption band or visible wavelength [1-3], and hence can be a critical hurdle to overcome in the Yb-doped fibre (YDF) based devices. It is generally recognised that the photodarkening induces excess background loss at the Yb-band, and this leads to degradation of the device performance. Here we report the influence of the photodarkening at a pump wavelength to envisage the direct impact of the photodarkening in the YDF based devices. Our results suggest that the photodarkening does not only induce excess background loss as commonly interpreted, but also influences pump efficiency.

A $1 \mathrm{~cm}$ long YDF with Al: $\mathrm{Yb}$ core composition was pumped at $977 \mathrm{~nm}$ and the transmitted power at 977 and $633 \mathrm{~nm}$ was monitored over time. The $\mathrm{Yb}$ concentration in the fibre was approximated to be 15,000 ppm-wt. Figure 1(a) shows the temporal change of transmission at both wavelengths when $46 \%$ of $\mathrm{Yb}$ ions are excited. The transmission at the wavelengths can be characterised by an exponential decay curve as reported in [2]. A saturated induced loss is estimated as $1695 \mathrm{~dB} / \mathrm{m}$ at $633 \mathrm{~nm}$ and $89 \mathrm{~dB} / \mathrm{m}$ at $977 \mathrm{~nm}$. The results indicate that $\sim 20 \%$ of pump power is wasted due to the photodarkening at the given experimental condition. This amount of pump power reduction is critical to device performance. Interestingly, the ratio of induced loss between the monitored wavelengths is $\sim 19$ which is much smaller than the known value of $\sim 70$ when measured from the transmitted power spectrum after the pumping [1]. The transmitted power spectrum can only show an induced background loss whereas our measurement directly reveals the reduction of pump power caused by the photodarkening. The revealed larger pump power loss can suggest that the pump power is utilized to create defect centres accounting for the photodarkening [4]. In addition to the pump power reduction, fluorescence decay time is observed to reduce by $12 \%$ after the photodarkening. As the lifetime linearly scales to laser efficiency [5], lasers operating at $46 \%$ of population inversion will carry $12 \%$ performance degradation over time. Thus, this can additionally hamper the device performances.

An effect of the pump power reduction due to the photodarkening is investigated in an YDF amplifier under a counter-pumping configuration. The seed input power and wavelength was varied in the experiments while pump power was fixed at $185 \mathrm{~mW}$. Figure 1(b) shows the signal power drop at the various conditions of the signal seed. The output signal power drops $\sim 80 \%$ when the amplifier operates in an unsaturated regime and $\sim 65 \%$ in a saturated regime. The results are consistent with the excited population density over the YDF. Thus, our results propose that operating in saturated regime is anticipated for long term reliability of the device. Furthermore, we noticed from our calculation that pump efficiency reduces by $15 \sim 30 \%$ in our amplifiers due to the photodarkening.

We also explored a pre-annealing of Yb-doped preform to control the photodarkening. The results suggest the precursor of the photodarkening can be thermally active.
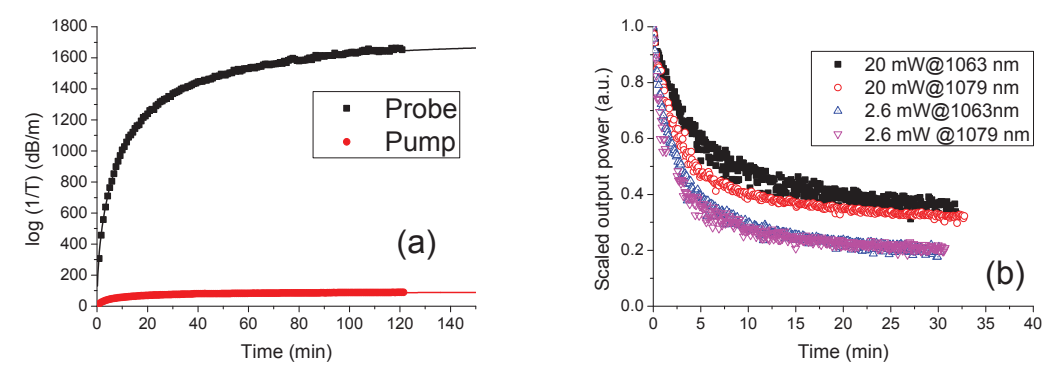

Fig. 1 (a) Change of transmission at 633 and $976 \mathrm{~nm}$ (b)Signal power drop in Yb-doped fibre amplifier due to photodarkening

\section{References}

[1] J. J. Koponen, M. J. Soderlund, H. J. Hoffman, and S. K. T. Tammela, "Measuring photodarkening from single-mode ytterbium doped silica fibers," Opt. Express 14, 11539 (2006).

[2]S. Jetschke, S. Unger, U. Ropke, and J. Kirchhof, "Photodarkening in Yb doped fibers: experimental evidence of equilibrium states depending on the pump power," Opt. Express 15, 14838 (2007).

[3] S. Yoo, C. Basu, A. J. Boyland, C. Sones, J. Nilsson, J. K. Sahu, and D. Payne, "Photodarkening in Yb-doped aluminosilicate fibers induced by $488 \mathrm{~nm}$ irradiation," Opt. Lett. 32, 1626 (2007).

[4] T. Arai, K. Ichii, K. Okada, T. Kitabayashi, S. Tanigawa, and M. Fujimaki, "Photodarkening phenomenon in Yb-doped fibers," Fujikura Technical Review, 6 (2009).

[5] J. D. Prohaska, D. P. Machewirth, and E. Snitzer, "Quenching of neodymium fluorescence by molcular-hydrogen," Opt. Lett. 20, 719 (1995). 